\title{
Calprotectin (S100A8/S100A9): a key protein between inflammation and cancer
}

\author{
Fatemeh Shabani ${ }^{1} \cdot$ Alireza Farasat $^{2} \cdot$ Majid Mahdavi $^{1} \cdot$ Nematollah Gheibi $^{2}(\mathbb{0}$
}

Received: 17 May 2018 / Revised: 19 July 2018 / Accepted: 25 July 2018

(c) Springer Nature Switzerland AG 2018

\begin{abstract}
Background Calprotectin (S100A8/S100A9), a heterodimeric EF-hand $\mathrm{Ca}^{2+}$ binding protein, are abundant in cytosol of neutrophils and are involved in inflammatory processes and several cancerous pathogens.

Objective The purpose of the present systematic review is to evaluate the pro- and anti-tumorigenic functions of calprotectin and its relation to inflammation.

Materials and methods We conducted a review of studies published in the Medline (1966-2018), Scopus (2004-2018), ClinicalTrials.gov (2008-2018) and Google Scholar (2004-2018) databases, combined with studies found in the reference lists of the included studies.

Results Elevated levels of S100A8/S100A9 were detected in inflammation, neoplastic tumor cells and various human cancers. Recent data have explained that many cancers arise from sites of infection, chronic irritation, and inflammation. The inflammatory microenvironment which largely includes calprotectin, has an essential role on high producing of inflammatory factors and then on neoplastic process and metastasis.

Conclusion Scientists have shown different outcomes in inflammation, malignancy and apoptosis whether the source of the aforementioned protein is extracellular or intracellular. These findings are offering new insights that anti-inflammatory therapeutic agents and anti-tumorigenic functions of calprotectin can lead to control cancer development.
\end{abstract}

Keywords Calprotectin $\cdot$ S100A8/S100A9 $\cdot$ Inflammation $\cdot$ Cancer $\cdot$ Apoptosis

\section{Introduction}

The S100s are low molecular weight proteins which represent the largest subgroup within the $\mathrm{Ca}^{2+}$-binding EF-hand. This superfamily comprising of more than 20 members in the human genome, it's genes cluster are located on chromosome 1q21 and similar numbers have been found in other mammalian based on genomic analysis [1]. They were first isolated originally from bovine brain tissue by Moore et al. in 1965 and represent small acidic proteins with a unique solubility in a solution of $100 \%$ saturated ammonium sulfate,

Responsible Editor: John Di Battista.

Nematollah Gheibi

ngheibi@qums.ac.ir

1 Department of Biochemistry, University of Tabriz, Tabriz, Iran

2 Cellular and Molecular Research Center, Qazvin University of Medical Sciences, Qazvin, Iran which termed "S100" $[2,3]$. Calprotectin, a members of S100s proteins, is a heterodimeric calcium-binding protein consisting of S100A8 and S100A9 subunits [4]. They are expressed in a wide variety of cell types and are abundant in myeloid cells such as: neutrophils, monocytes, keratinocytes and early differentiation states of macrophages. So, S100A8 and S100A9 are called myeloid-related protein and are known as: MRP-8 and MRP-14 too [5, 6]. Furthermore, S100A8 and S100A9 are denoted as Calgranulin A and Calgranulin $\mathrm{B}$ respectively which reflect their calcium-binding properties and highly overexpressed in granulocytes. The S100A8/S100A9 are released by activated granulocytes and act in a cytokine-like manner by binding to cell surface receptors which trigger signaling pathways involved in the inflammatory processes and play critical roles in numerous cellular processes includes cell cycle progression, cell survival, proliferation, differentiation, and cell migration. Furthermore, they exert a wide range of functions including: calcium homeostasis regulation which involved in proteins phosphorylation, cytoskeletal components rearrangement, 